\title{
Immunonutrition in perioperative phase: An insight into the integrated treatment of cardiac surgery patients
}

\author{
De Santis $\mathrm{L}^{1 *}$, Maio $\mathrm{D}^{1}$, Cavone $\mathrm{M}^{1}$, Serlenga $\mathrm{E}^{2}$ and Minelli $\mathrm{M}^{1}$ \\ ${ }^{1}$ Dipartimento di Immunologiaclinica e Medicina Personalizzata, GVM Care and Research, Italy \\ ${ }^{2}$ Servizio Immunotrasfusionale Azienda Sanitaria Locale BAT, Italy
}

\begin{abstract}
The clinical condition of a patient scheduled for surgery, in addition to being a crucial point for the positive result of the operation, is certainly able to affect also the post-surgery phase.For this reason, the patient needs to be evaluated globally and in an interdisciplinary way in order to limit eventual early or late complications after surgery as much as possible. In the last few years, the knowledge gained through the progress and developments of basic research aimed at assessing the effects of some principles having immunostimulant properties, has allowed a clearer understanding of the role of the gut and of the gut-associated lymphoid tissue (GALT) in the immunologic dynamics of protection of the body against extraneous pathogens and agents that regulate the tolerance to commensal bacteria and food antigens.
\end{abstract}

\section{Introduction}

The clinical condition of a surgical patient takes on a fundamental relevance both in the perspective of a positive outcome of the intervention planned and of a rapid evolution of the post surgery progress. On the other hand, since the status of health of the subject scheduled for surgery cannot be referred only to the standards of a hematochemical routine, it needs to be carefully re-examined in an integral and systemic mode and eventually brought back to a condition of balanced organic homeostasis and overall clinical stabilization.

The depression in cellular immunity associated with the surgical event is well known [1], and is ascribable mostly to functional alterations in the immune system associated with surgical stress and basically related to a shift in the physiological homeostatic balance between proinflammatory and regulatory cytokines. The possibility that this may occur can account for an increase in post surgery complications and in mortality [2].

Examining the patient as a whole means assessing also his nutritional status through appropriate screening tests including, targeted hematochemical examinations, study of body composition by means of anthropometry and bioimpedancemetry, with the aim of limiting early or late postoperative complications as much as possible [3].

\section{Protein-energy malnutrition}

It is well known that protein deficiency has the capacity to considerably weaken the immune response, thus increasing the susceptibility to infections. Actually, protein-energy malnutrition seems to be the leading cause of immunodeficiency worldwide [3].

Although the protein intake required for a proper function of the Immune System is already widely known, research on the use of single amino acids as a dietary support aimed at optimizing the immune function has only recently begun. The first studies show that amino acids constitute an important energy substrate for immune system cells
[4] as well as for antioxidant defence mechanisms [5].

Moreover, in certain pathological conditions, such as burns, traumas, total parenteral nutrition, or in physiological status during pregnancy and weaning, a number of non-essential amino acids (arginine, glutamine, glutamate, glycine, proline, taurine, and cysteine) have been found to become compulsorily essential. This change in the need for specific amino acids can be due in part to their effects on the Immune System [6].

In 2010, Wu introduced a new concept of functional amino acids so as to better explain the role of those amino acids that had been defined simply non-essential until then. The importance of these organic molecules lies in their participation in regulating the main metabolic pathways aimed at enhancing organism health, survival, growth, development, lactation and reproduction [7]. Such metabolic pathways include: intracellular protein turnover and related events, synthesis and catabolism of amino acids, generation of small peptides, nitrogen metabolites and substances containing sulphur, urea cycle and synthesis of uric acid, lipid and glucose metabolism, metabolism of carbon units, redox cell signalling pathways [6].

Furthermore, malnutrition plays an important role also in increasing morbidity and mortality after heart surgery. Nonetheless, the nutritional assessment in patients before cardiac surgery can be somewhat demanding, for example, when considering the timing of the operation [8].

Nutritional screening, however, is an instrument that enables a rapid and effective identification of a high-risk patient. The nutritional condition of such patients, therefore, should be carefully studied

Correspondence to: De Santis L, Dipartimento di Immunologiaclinica e Medicina Personalizzata, GVM Care and Research, Italy, E-mail: ldesantis@nutrizioneperte.it

Received: June 10, 2015; Accepted: July 22, 2015; Published: July 25, 2015 
and should require, wherever necessary, a perioperative nutritional intervention.

Over the years, a number of screening tests have been developed, such as the Malnutrition Universal Screening Tool (MUST) [9] recommended in the European guidelines [10] for the nutritional assessment of adult population. The Nutritional Risk Screening (NRS) [11], instead, has been suggested for the detection of undernutrition and the risk of its development in patients while in hospital. Moreover, the Mini Nutritional Assessment (MNA) [12] is useful for the elderly receiving home care, or inpatients in hospitals and/or nursing homes. Finally, the Short Nutrition Assessment Questionnaire (SNAQ) has been developed for outpatients.

Malnutrition is a widespread condition among patients with cardiovascular diseases and is related to adverse postoperative events. Moreover, malnutrition occurs in $10-25 \%$ of cardiac surgery patients [13]. However, in spite of the identification of the specific mechanisms of malnutrition development in patients with congestive cardiac insufficiency leading to cardiac cachexia, a specific tool for nutritional assessment in this kind of population has not been designed so far [14].

In 2003, a comparative study examined the various nutritional screening tests with the aim to assess the prognostic value of different screening tools for heart bypass surgery patients. The study showed that both MUST and MNA have independent predictive values regarding postoperative complications. Yet, taking into account the uncomplicated use of the MUST, this tool is to be preferred for heart surgery population [15].

\section{The role of the immune system}

The gut and gut-associated lymphoid tissue (GALT) are essential components of the Immune System capable of protecting the body from extraneous agents and pathogens and regulating the tolerance to commensal bacteria and food antigens [16].

The role of proteins in supporting, at different levels, the activity of the immune system has long been known. It is linked not only to protein intake, but also to the availability of specific amino acids (in particular glutamine, glutamate and arginine), which are essential for the enhancement of the immune functions of the gut and of resident immune cells [6].

Each of these amino acids has unique properties including maintenance of membrane integrity, gut increase and function, as well as regulation of inflammatory cytokine secretion, increase in the number of $\mathrm{T}$ lymphocytes, improvement of specific $\mathrm{T}$ cells function, and secretion of IgAs by the cells in the lamina propria [16].

The Immune System, following surgery, can undergo a change in the ratio between peripheral blood mononuclear cells, thus increasing prostaglandin E2 synthesis by monocytes and simultaneously decreasing T CD3-CD4+ lymphocyte function.

As already known, T cells can be subdivided into T helper 1 (Th1) and $\mathrm{T}$ helper 2 (Th2), each group capable of synthesizing distinct cytokine profiles. Th 1 cells produce IFN- $\gamma$, IL- 2 and IL- 12 interferon, fostering cell-mediated immunity, whereas Th2 cells produce IL-4, IL-6, IL-10 and IL-13, promoting humoral immunity [17]. It has been shown that, after cardiac surgery, the levels of IL- 2 and IFN- $\gamma$ decrease and, as a result, also Th1 activity is reduced, while IL- 4 and IL-10 levels increase and, as a consequence, Th2 activity increases as well.

The influence of preoperative supplementation on the key parameters of inflammation has been widely studied. By 1995 , Wachtler et al. had studied the effect of preoperative supplementation on postoperative levels of leukotrienes derived from fatty acids, revealing that the group of patients treated had generated more antiinflammatory leukotrienes to the detriment of pro-inflammatory leukotrienes [18].

However, no effect on the proliferation of lymphocytes, or on monocyte function, was observed, probably due to the fact that no group reached sufficient arginine levels through supplementation. Tepaske et al. analyzed the efficacy of immunonutrition in improving the host defence during the preoperative phase, and in reducing organ infections and dysfunctions in the postoperative period. The group under treatment showed an increase in the host defences before surgery, through a significant increase in the expression of HLA-DR epitopes on monocytes. Moreover, IL- 6 concentration showed a decrease, while delayed hypersensitivity response to antigens improved and persisted until discharge from hospital [19].

The above mentioned, as well as other works, show how supplementation with immunonutrients can improve the immune function and limit the inflammatory response after surgery. In addition, the immunomodulating effects of these nutritional supports contribute to preventing organ damage after surgery and improve organ reperfusion. Moreover, the overall improvement of the nutritional status has been shown to reduce the rate of postoperative complications and organ dysfunction.

\section{Immunonutrients}

\section{Arginine}

Arginine is a non-essential amino acid in adults, since it can be synthesized starting from glutamine, glutamate and proline, yet, it becomes indispensable under stress [20,21].

On the contrary, this amino acid proves to be essential for children and adolescents. It has to be taken in through nutrition since the body is unable to synthesize it in sufficient quantity to support normal growth. Indeed, a number of studies conducted on piglets have shown that arginine supplementation fosters growth [22].

Effects of arginine on intestinal function: Arginine is the only nitric oxide precursor synthase (all isoforms) for the synthesis of nitric oxide, which is essential for an optimally functioning Immune System, including regulation of the inflammatory response [23].

As above mentioned, intestinal structure and function have shown to be sensitive to the quantity of arginine contained in food and in relation to pathologic status or stress. Several studies have shown that supplementation with arginine from 0.6 to $1 \%$ in relation to weight leads to an improvement on the histology of intestinal mucosa, particularly on the height of villi and the depth of the crypts. A mechanism proposed for such evidence is that arginine might increase the HSP 70 (Heat Shock Protein 70 kilodaltons) expression, which in its turn prevents protein denaturation and, as a consequence, the cell stress associated with it [24]. In particular, HSP70 cells help the other nascent cells reach the definitive spatial structure and foster cell protection against stress factors.

Moreover, other researchers have shown that arginine intake $(0.33$ g daily) improves the intestinal barrier function [25].

Finally, the lack of arginine in diet has been shown to be associated 
with other conditions such as reproductive, metabolic and neurologic disorders [21].

Effects of arginine on the immune system: The immunomodulating effects of arginine have already been explained [26,27], and its role in innate as well as in adaptive immunity has been widely demonstrated. In particular, arginine is fundamental for nitric oxide synthase activity (see above), but also for B cell development and T-cell receptor activity [20].

Other studies conducted on rats affected by acute pancreatitis have shown that supplementation with arginine induces an increase in T-helpers and a higher ratio of $\mathrm{CD} 4 / \mathrm{CD} 8+$ in the intestinal cells of the lamina propria [28].

By assessing the cytokine profile of rats that received arginine integration, it was possible to observe a decrease in proinflammatory cytokines such as IFN- $\gamma$ and IL-2, and an increase in IL-4 and IL-10 with anti-inflammatory property [29].

\section{Glutamine}

Glutamine is a non-essential amino acid that is present in large quantities in the human body, and is an important energy substrate as precursor of other amino acids. However, under stress and during critical developmental phases, the essentiality of exogenous sources of glutamine has already been established [6].

In the cells of the immune system, particularly in lymphocytes, neutrophils and macrophages, glutamine is rapidly utilized and metabolized to glutamate, aspartate, lactate and $\mathrm{CO}_{2}$.

Moreover, being a precursor of glutamate, glutamine enhances the production of glutathione (GSH), an important regulator of redox in enterocytes and lymphocytes [30].

This amino acid provides nitrogen for the synthesis of nucleic acids and proteins that are essential to the proliferation of lymphocytes and for the production of signals such as cytokines [31].

Effects of glutamine on the immune system: The importance of glutamine for the function of B- and T-lymphocytes, neutrophils and macrophages has been shown both in vitro and in vivo [32].

A number of studies have shown that glutamine supplementation induces a decrease in the inflammatory cytokine level and an increase in the response of proliferating cells, MNL, and B and T cells.

Moreover, further studies have shown that a glutamine supplement is required in the amount of $0.5-5 \%$ body weight in order to maintain a healthy intestinal mucosa and support several GALT functions.

\section{Fatty acids}

There are two main families of polyunsaturated fatty acids: $\omega-3$ fatty acids, comprising eicosapentaenoic acid (EPA) and docosahexaenoico acid (DHA) and $\omega-6$ fatty acids, including linoleic acid (LA), arachidonic acid (AA) and $\gamma$-linoleic acid.

Several studies have reported beneficial effects following preoperative administration of a supplement rich in $\omega-3$ fatty acids, arginine and RNA [33].

Moreover, preoperative administration of dietary supplement has been shown to improve preoperative nutritional status and pre- and postoperative immune response [4].

Further recent studies have concluded that administration of dietary supplement rich in $\omega-3$ fatty acids, arginine and RNA can inhibit inflammatory and thrombotic responses in the initial phase after cardiac surgery and, therefore, decrease the incidence of postoperative complications $[34,35]$.

\section{Oxidative stress and cardiovascular diseases}

Reperfusion, that is restoration of blood circulation in a tissue after a period of ischemia, e.g. following occlusion of a coronary artery later re-canalized, is associated with conditions of oxidative stress that can produce considerable tissue damage.

This condition assumes particular importance in widespread chronic pathologies such as myocardiopathy and ischemic encephalopathy, and also contributes to determine kidney tissue damage observable in a number of districts in patients who have suffered from severe and prolonged shock.

Moreover, what above stated acquires particular importance also in transplant medicine, where, by definition, an organ undergoes ischemic conditions after the removal from the donor, and reperfusion while being reconnected to the recipient circulation.

During the period of ischemia, moderate increased levels of ROS, originating from mitochondrial respiration, can already be observed. The massive release of ROS during reperfusion, instead, originates for different reasons and can derive from two mechanisms: 1) activation of xanthine oxidase, following accumulation in the tissue of purine catabolites of ATP consumed during anoxic phase, entails a massive intracellular production of superoxide anion and hydrogen peroxide; 2) the adherence of neutrophilic granulocytes (during reperfusion) on the endothelium injured during the ischemic phase, is associated with their activation, thus producing high levels of, in this case extracellular, pro-oxidants [36].

The high production of ROS, on the other hand, is capable of modifying also the subcellular compartmentation of calcium ions. In the myocardial cell, lipid peroxidation of the sarcoplasmic membrane causes inhibition of Ca++-ATPase and release of calcium in the cytoplasm. The direct oxidation of the $\mathrm{SH}$ groups of the enzyme by the superoxide anion or hydrogen peroxide has asimilar effect. On the other hand, ROS and lipidic peroxidation products cause uncoupling of mitochondrial oxidative phosphorylation, thus altering $\mathrm{Ca}++$ transportation also in that site. The increased cytoplasmic levels of $\mathrm{Ca}++$ seem to serve as common denominator in a number of pathologic processes in the cardiovascular district, such as intimal hyperplasia in the initial stage of atherosclerosis, phenomena of vasoconstriction linked to increased artery pressure, myocardial ischemia-reperfusion injury, and hypertrophy that signals heart failure [37].

\section{Antioxidant action of vitamins $\mathrm{C}$ and $\mathrm{E}$}

Vitamin C, or ascorbic acid, is a nutrient required for a variety of biologic functions and has its biologic effects mainly by acting as an enzymatic cofactor and as an antioxidant.

Human beings, primates and a few other animals (e.g. guinea pigs) depend on diet for the intake of vitamin $\mathrm{C}$ so as to avoid deficiencyrelated diseases, such as scurvy, and maintain good general health. The healthy effects of vitamin $\mathrm{C}$ can be ascribed to its biological functions as a cofactor for a certain number of enzymes, particularly hydroxylases involved in collagene synthesis, and as a hydrosoluble antioxidant.

The reactive oxygen species (ROS), produced by a variety of sources, such as the electron transport chain, xanthine oxidase, myeloperoxidase 
and NADPH oxidase, start a radical reaction. Lipids are often the main targets of oxygen radicals, since many enzymes that produce ROS are incorporated into lipid bilayers, and lipid peroxidation can be seen as an example of radical chain reaction. Lipid radicals react with molecular oxygen, thereby creating peroxyl radicals that, if they are not neutralized by $\alpha$-tocopherol (Vitamin E) into membranes, can take part in the radical propagation reaction. Lipid hyperoxids are chemically unstable compounds that, when not reduced by glutathione reductase to form hydroxy fatty acids, constitute a source for a variety of products, among which LPO alkenals, epoxides and malondialdehyde. Vitamin C has the capacity to protect against the formation of these products by acting as ROS scavengers and reducing lipid hydroperoxyl radicals by one electron via the Vitamin E redox cycle [38].

\section{Vitamin D}

An increasing body of evidences suggest that low Vitamin D levels have negative effects on cardiovascular health, includingcoronary risk and adverse cardiovascular outcomes such as myocardial infarction, heart failure and stroke. There is some evidence suggesting that a higher percentage of individuals with cardiovascular diseases have low Vitamin D levels when compared with the general population. Some researchers have studied the prevalence of Vitamin D deficiency in heart surgery patients and test results revealed that $92.5 \%$ of patients had Vitamin D levels $<75 \mathrm{nmol} / \mathrm{L}, 67.5 \%$ had levels $<60 \mathrm{nmol} / \mathrm{L}, 52.5 \%$ had levels between 30-59 nmol/L and 15\% had levels $<30 \mathrm{nmol} / \mathrm{L}$.

Moreover, inadequate Vitamin D levels were found in $80 \%$ of obese patients (BMI > $30 \mathrm{Kg} / \mathrm{m}$ compared to $59 \%$ of non-obese patients.

Therefore, two-thirds of patients had serum Vitamin D levels below $60 \mathrm{nmol} / \mathrm{L}$, placing them at higher risk of relapses that, in their turn, are associated with a high mortality risk. The researchers concluded that in an ageing population with CVD, Vitamin D status needs to be assessed and any inadequacy corrected [39].

\section{Selenium}

Selenium is an essential nutritional element for mammals necessary for the function of a number of oxidant enzymes, such as glutathione peroxidase $(\mathrm{GPx})$, thioredoxin reductase (TrxR) and iodothironine deiodinase (IDD). The antioxidant effect of selenium is essential to human physiology since the oxidative stress is associated with several pathologies such as cardiovascular diseases, diabetes, or cancer, and is also associated with most of surgical procedures.

In particular, the use of cardiopulmonary bypass for cardiac surgery is always related to oxidative stress that causes ischemia and reperfusion.

The pathogenesis of the damage after ischemia/reperfusion depends on many factors, among which the formation of reactive nitrogen species (RNS) and reactive oxygen species (ROS), seen as being the cause of the lesion. ROS formed during the oxidative stress can start lipid peroxidation and protein oxidation, thus causing damages to DNA. The production of ROS is physiologically controlled by free radical "scavengers", such as GPx and TrxR, and SOD (superoxide dismutase). GPx and TrxR are seleno-cisteine dependent enzymes and their activity is known to be related to selenium availability. Furthermore, selenium has been suggested in the regulation of the gene expression of such selenoproteins as a cofactor, and there are also evidences that selenium supplementation may reduce the oxidative stress and decrease complications after cardiac surgery. However, other clinical studies have not demonstrated an association between selenium deficiency and cardiovascular results [36].

\section{Probiotics and immune-mediated activities}

\section{Lactobacillus rhamnosus}

Recent literature shows evidence for the immunomodulatory effects of probiotics, mediated in part by regulatory $\mathrm{T}$ helper cells, which on the one hand increase inflammatory cytokine expression, such as IL-10, and on the other decrease proinflammatory cytokine expression, such as INF- $\gamma$, TFN- $\alpha$ and IL-12 [37]. Moreover, there are interesting results from a study showing evidence of a decrease in the rate of incidence of allergic sensitization in subjects with colonization of the gastrointestinal tract by gram-positive bacteria in the gut flora, among which also lactobacilli and bifidobacteria [38,39].

Based on these early works, it was possible to assess the efficacy of the use of probiotics to treat allergic disorders. The first study was conducted by Kalliomaki et al. [40-44]. This clinical trial, conducted on pregnant women, involved administration of Lactobacillus rhamnosus strain GG (LGG) to women with a family history of allergic pathology in their own families or in their partners', 2-4 weeks before the end of pregnancy and for 6 months after delivery. During the second year of follow-up, a 50\% reduction in the rate of incidence of atopic dermatitis was observed in the treatment group compared to the control group. Such beneficial effects, however, did not extend to the fifth year of followup. In the most recent study in this field, Wickens et al. [45] showed evidence of a different effect in the prevention of atopic dermatitis by using Lactobacillus rhamnosus and Bifidobacterium animalis subsp. lactis. The results revealed that perinatal supplementation with Lactobacillus rhamnosus significantly reduced the risk of dermatitis by circa $50 \%$ compared to the placebo group.

Some probiotics may regulate apoptosis (the so-called "programmed death") of the intestinal epithelial cells (IEC), among which Lactobacillus rhamnosus GG, capable of activating an antiapoptotic action protein and of inhibiting a pro-apoptotic action protein on IECs stimulated by a number of cytokines (TNF- $\alpha$, IL$1 \alpha-\beta$ or IFN $\gamma$ ). Other trials indicate, moreover, that LGG activates the production of two proteins, p75 and p40, which promote cell proliferation and anti-apoptotic Akt protein activation [46-50].

\section{Discussion}

In the last few years, the role of some nutrients in modulating different types of disorders has become increasingly evident. Such organic molecules, generally defined as immunonutrients, include some amino acids, essential fatty acids and probiotics.

Among immunomodulatory amino acids, arginine, the only precursor of nitric oxide synthase, is essential for an optimal function of the Immune System, since it regulates the inflammatory response by reducing proinflammatory cytokines production and increasing antiinflammatory cytokines. Furthermore, it enhances intestinal barrier function, and the lack of arginine from diet is implied in reproductive, metabolic and neurologic disorders.

Glutamine, on the other hand, plays its immunomodulatory role by providing an oxidative substrate for immune cells and reducing proinflammatory cytokine levels.

A great number of studies confirm also the immunomodulatory action of Lactobacillus rhamnosus that performs its efficacy in controlling, for example, atopic dermatitis. Moreover, it has also 
produced important results in the treatment of acute infectious diarrhoea in children, in the prevention of diarrhoea from antibiotics, in the prevention of nosocomial diarrhoea in children, as a coadjuvant therapy for H. pylori eradication and in reducing symptoms of irritable colon syndrome.

Finally, the capacity of probiotics to regulate apoptosis can represent a useful strategy in controlling intestinal infections.

\section{References}

1. Ni Choileain N, Redmond HP (2006) Cell response to surgery. Arch Surg 141: 11321140. [Crossref]

2. Strohmeyer JC, Blume C, Meisel C, Doecke WD, Hummel M, et al. (2003) Standardized immune monitoring for the prediction of infections after cardiopulmonary bypass surgery in risk patients. Cytometry B Clin Cytom 53: 54-62. [Crossref]

3. Field CJ, Johnson IR, Schley PD (2002) Nutrients and their role in host resistance to infection. J Leukoc Biol 71: 16-32. [Crossref]

4. Field CJ, Wu G, Marliss EB (1994) Enhanced metabolism of glucose and glutamine in mesenteric lymph node lymphocytes from spontaneously diabetic BB rats. Can $J$ Physiol Pharmacol 72: 827-832. [Crossref]

5. Xue H, Field CJ (2011) New role of glutamate as an immunoregulator via glutamate receptors and transporters. Front Biosci (Schol Ed) 3: 1007-1020. [Crossref]

6. Wu G (2013) Functional amino acids in nutrition and health. Amino Acids 45: 407-411. [Crossref]

7. Wu G, Bazer FW, Burghardt RC, Johnson GA, Kim SW, et al. (2010) Impacts of amino acid nutrition on pregnancy outcome in pigs: mechanisms and implications for swine production. J Anim Sci 88: E195-204. [Crossref]

8. Sanchez JA, Sanchez LL, Dudrick SJ (2011) Nutritional considerations in adult cardiothoracic surgical patients. Surg Clin North Am 91: 857-875, ix. [Crossref]

9. Kondrup J, Allison SP, Elia M, Vellas B, Plauth M; Educational and Clinical Practice Committee, et al. (2003) ESPEN guidelines for nutrition screening 2002. Clin Nutr 22: 415-421. [Crossref]

10. Malnutrition Advisory Group (MAG). MAG-Guidelines for detection and management of malnutrition. Redditch, UK: British Association for Parenteral and Enteral Nutrition, 2000.

11. Kondrup J, Rasmussen HH, Hamberg O, Stanga Z; Ad Hoc ESPEN Working Group (2003) Nutritional risk screening (NRS 2002): a new method based on an analysis of controlled clinical trials. Clin Nutr 22: 321-336. [Crossref]

12. Vellas B, Guigoz Y, Garry PJ, Nourhashemi F, Bennahum D, et al. (1999) The Min Nutritional Assessment (MNA) and its use in grading the nutritional state of elderly patients. Nutrition 15: 116-122. [Crossref]

13. van Venrooij LM, van Leeuwen PA, de Vos R, Borgmeijer-Hoelen MM, de Mol BA (2009) Preoperative protein and energy intake and postoperative complications in wellnourished, non-hospitalized elderly cardiac surgery patients. Clin Nutr 28: 117-121. [Crossref]

14. Anker SD, Coats AJ (1999) Cardiac cachexia: a syndrome with impaired survival and immune and neuroendocrine activation. Chest 115: 836-847. [Crossref]

15. Lomivorotov VV, Efremov SM, Boboshko VA, Nikolaev DA, Vedernikov PE, et al. (2013) Prognostic value of nutritional screening tools for patients scheduled for cardiac surgery. Interact Cardiovasc Thorac Surg 16: 612-618. [Crossref]

16. Ruth MR, Field CJ (2013) The immune modifying effects of amino acids on gutassociated lymphoid tissue. J Anim Sci Biotechnol 4: 27. [Crossref]

17. Romagnani S (2000) T-cell subsets (Th1 versus Th2). Ann Allergy Asthma Immunol 85: 9-18. [Crossref]

18. Wachtler P, Axel Hilger R, König W, Bauer KH, Kemen M, et al. (1995) Influence of a pre-operative enteral supplement on functional activities of peripheral leukocytes from patients with major surgery. Clin Nutr 14: 275-282. [Crossref]

19. Tepaske R, Velthuis H, Oudemans-van Straaten HM, Heisterkamp SH, van Deventer SJ, et al. (2001) Effect of preoperative oral immune-enhancing nutritional supplement on patients at high risk of infection after cardiac surgery: a randomised placebocontrolled trial. Lancet 358: 696-701. [Crossref]

20. Li P, Yin YL, Li D, Kim SW, Wu G (2007) Amino acids and immune function. $\mathrm{Br} J$ Nutr 98: 237-252. [Crossref]
21. Wu G (2009) Amino acids: metabolism, functions, and nutrition. Amino Acids 37: 1-17. [Crossref]

22. Wu X, Ruan Z, Gao Y, Yin Y, Zhou X, et al. (2010) Dietary supplementation with L-arginine or N-carbamylglutamate enhances intestinal growth and heat shock protein-70 expression in weanling pigs fed a corn- and soybean meal-based diet. Amino Acids 39: 831-839. [Crossref]

23. Nakamura H, Kawamata Y, Kuwahara T, Torii K, Sakai R (2013) Nitrogen in dietary glutamate is utilized exclusively for the synthesis of amino acids in the rat intestine. $\mathrm{Am}$ J Physiol Endocrinol Metab 304: E100-108. [Crossref]

24. Yao K, Guan S, Li T, Huang R, Wu G, et al. (2011) Dietary L-arginine supplementation enhances intestinal development and expression of vascular endothelial growth factor in weanling piglets. Br J Nutr 105: 703-709. [Crossref]

25. Ersin S, Tuncyurek P, Esassolak M, Alkanat M, Buke C, et al. (2000) The prophylactic and therapeutic effects of glutamine- and arginine-enriched diets on radiation-induced enteritis in rats. $J$ Surg Res 89: 121-125. [Crossref]

26. Field CJ, Johnson I, Pratt VC (2000) Glutamine and arginine: immunonutrients for improved health. Med Sci Sports Exerc 32: S377-388. [Crossref]

27. Popovic PJ, Zeh HJ 3rd, Ochoa JB (2007) Arginine and immunity. J Nutr 137: 1681S-1686S. [Crossref]

28. Qiao SF, Lu TJ, Sun JB, Li F (2005) Alterations of intestinal immune function and regulatory effects of L-arginine in experimental severe acute pancreatitis rats. World $J$ Gastroenterol 11: 6216-6218. [Crossref]

29. Fan J, Meng Q, Guo G, Xie Y, Li X, et al. (2010) Effects of early enteral nutrition supplemented with arginine on intestinal mucosal immunity in severely burned mice. Clin Nutr 29: 124-130. [Crossref]

30. Wu G, Fang YZ, Yang S, Lupton JR, Turner ND (2004) Glutathione metabolism and its implications for health. J Nutr 134: 489-492. [Crossref]

31. Calder PC, Yaqoob P (1999) Glutamine and the immune system. Amino Acids 17: 227 241. [Crossref]

32. Newsholme $\mathrm{P}$ (2001) Why is L-glutamine metabolism important to cells of the immune system in health, postinjury, surgery or infection? J Nutr 131: 2515S-22S. [Crossref]

33. Senkal M, Kemen M, Homann HH, Eickhoff U, Baier J, et al. (1995) Modulation of postoperative immune response by enteral nutrition with a diet enriched with arginine, RNA, and omega-3 fatty acids in patients with upper gastrointestinal cancer. Eur J Surg 161: 115-122. [Crossref]

34. Nakamura K, Moriyama Y, Kariyazono H, Hamada N, Toyohira H, et al. (1999) Influence of preoperative nutritional state on inflammatory response after surgery. Nutrition 15: 834-841. [Crossref]

35. Iwase H, Kariyazono H, Arima J, Yamamoto H, Nakamura K (2014) Nutritional Effect of Oral Supplement Enriched in $\omega-3$ Fatty Acids, Arginine, RNA on Immune Response and Leukocyte-platelet Aggregate Formation in Patients Undergoing Cardiac Surgery. Nutr Metab Insights 7: 39-46. [Crossref]

36. Jordan JE, Zhao ZQ, Vinten-Johansen J (1999) The role of neutrophils in myocardial ischemia-reperfusion injury. Cardiovasc Res 43: 860-878. [Crossref]

37. Dhalla NS, Golfman L, Takeda S, Takeda N, Nagano M (1999) Evidence for the role of oxidative stress in acute ischemic heart disease: a brief review. Can J Cardiol 15 . 587-593. [Crossref]

38. Traber MG, Stevens JF (2011) Vitamins C and E: beneficial effects from a mechanistic perspective. Free Radic Biol Med 51: 1000-1013. [Crossref]

39. Braun LA, Spitzer O, Levkovich B, Bailey M, Stanguts C2, et al. (2014) Prevalence of vitamin D deficiency prior to cardiothoracic surgery. Heart Lung Circ 23: 978-980. [Crossref]

40. Guo F, Monsefi N, Moritz A, Beiras-Fernandez A (2012) Selenium and cardiovascular surgery: an overview. Curr Drug Saf 7: 321-327. [Crossref]

41. Pessi T, Sütas Y, Hurme M, Isolauri E (2000) Interleukin-10 generation in atopic children following oral Lactobacillus rhamnosus GG. Clin Exp Allergy 30: 1804-1808. [Crossref]

42. Björkstén B (1999) The intrauterine and postnatal environments. J Allergy Clin Immunol 104: 1119-1127. [Crossref]

43. Kalliomäki M, Kirjavainen P, Eerola E, Kero P, Salminen S, et al. (2001) Distinct patterns of neonatal gut microflora in infants in whom atopy was and was not developing. J Allergy Clin Immunol 107: 129-134. [Crossref] 
44. Kalliomäki M, Salminen S, Arvilommi H, Kero P, Koskinen P, et al. (2001) Probiotics in primary prevention of atopic disease: a randomised placebo-controlled trial. Lancet 357: 1076-1079. [Crossref]

45. Rautava S, Kalliomäki M, Isolauri E (2002) Probiotics during pregnancy and breastfeeding might confer immunomodulatory protection against atopic disease in the infant. J Allergy Clin Immunol 109: 119-121. [Crossref]

46. Kalliomäki M, Salminen S, Poussa T, Arvilommi H, Isolauri E (2003) Probiotics and prevention of atopic disease: 4-year follow-up of a randomised placebo-controlled trial. Lancet 361: 1869-1871. [Crossref]

47. Kalliomäki M, Salminen S, Poussa T, Isolauri E (2007) Probiotics during the first 7 years of life: a cumulative risk reduction of eczema in a randomized, placebo-controlled trial. J Allergy Clin Immunol 119: 1019-1021. [Crossref]

48. Yan F, Cao H, Cover TL, Whitehead R, Washington MK, et al. (2007) Soluble proteins produced by probiotic bacteria regulate intestinal epithelial cell survival and growth. Gastroenterology 132: 562-575. [Crossref]

49. Wickens K, Black PN, Stanley TV, Mitchell E, Fitzharris P, et al. (2008) A differential effect of 2 probiotics in the prevention of eczema and atopy: a double-blind, randomized, placebo-controlled trial. J Allergy Clin Immunol 122: 788-794. [Crossref]

50. Yan F, Cao H, Cover TL, Whitehead R, Washington MK, et al. (2007) Soluble proteins produced by probiotic bacteria regulate intestinal epithelial cell survival and growth Gastroenterology 132: 562-575. [Crossref]

Copyright: (C2015 De Santis L. This is an open-access article distributed under the terms of the Creative Commons Attribution License, which permits unrestricted use, distribution, and reproduction in any medium, provided the original author and source are credited. 